\title{
Corrosion of cast Stellite-3 analogue in simulated PWR conditions
}

G.N. Karimi ${ }^{1}$, P.H. Shipway ${ }^{1}$, D.A. Stewart ${ }^{2}$ T. Hussain ${ }^{1}$

${ }^{1}$ Faculty of Engineering, University of Nottingham, University Park, Nottingham, NG7 2RD, UK

${ }^{2}$ Rolls-Royce plc, Derby, UK.

\section{Abstract}

Wear resistant alloys are required for deployment of pressurised water reactor primary circuits and in this context, the corrosion behaviour of a cast cobalt-based alloy following exposure for 30 days in lithiated water at $300^{\circ} \mathrm{C}$ was investigated. Corrosion of the surface was observed, resulting in the formation of a $\sim 100 \mathrm{~nm}$ thick chromium and silicon-rich oxide. Preferential corrosion of the matrix at its interface with just one of the carbide types was observed to a depth of $\sim 1 \mu \mathrm{m}$; for the first time this was found not due to any inhomogeneity in the matrix but was instead an electrochemical effect.

Keywords. Stellite, cobalt alloy, PWR hydrothermal corrosion, interfacial oxide, electrochemistry. 


\section{Introduction}

The cobalt-based alloys known as Stellites ${ }^{\circledR}$ (registered trade name of Kennametal Inc.) are very resistant to wear and are often the materials chosen for components where resistance to aggressive forms of corrosion and wear are required over a wide temperature, such as in valve seat hardfacings in the nuclear industry [1, 2]. These cobalt-based alloys are manufactured in a variety of ways such as casting, powder metallurgy (PM) (both with and without consolidation by hot isostatic pressing (HIP)), weld and laser hardfacing (using powder rod or wire consumables) and thermal spraying [3].

Stellite 3 has one of the highest carbon levels of the Stellites (2.0 - 2.4 wt.\% C), and thus a higher carbide fraction, with the carbides constituting about 30 wt. $\%$ of the material; the carbides are $\mathrm{M}_{7} \mathrm{C}_{3}$ (chromium-rich) and $\mathrm{M}_{6} \mathrm{C}$ (tungsten-rich) types [4, 5], with the $\mathrm{M}_{7} \mathrm{C}_{3}$ and the $\mathrm{M}_{6} \mathrm{C}$ being the primary and eutectic carbides formed in the solidification process respectively. Due to its high carbide fraction, it exhibits high wear and galling resistance (even when mated with other Stellites), but also some brittleness [6]. An investigation into the room temperature and high temperature $\left(450^{\circ} \mathrm{C}\right)$ sliding wear of various alloys showed that Stellite 3 exhibited higher wear resistance than Stellite $6(1.0-1.2 \mathrm{wt} . \% \mathrm{C})$ at both room and elevated temperature [7]. The alloy examined in the current study was a similar alloy to Stellite 3, namely the cobalt based cast alloy WT- $3^{\circledR}$ (Weartech International Ltd, UK) with a composition of Co $-29.31 \mathrm{Cr}-12.55 \mathrm{~W}-2.06 \mathrm{C}$ (wt.\%). The high tungsten content of $12.55 \mathrm{wt} . \%$ results in the precipitation of tungsten-rich carbide during alloy solidification [7].

Stellites are widely used for components subject to both wear and corrosion in the primary circuit of pressurised water reactors (PWR) in the nuclear industry. The 
primary circuit generally operates at temperatures between $280^{\circ} \mathrm{C}-320^{\circ} \mathrm{C}$ and a pressure of up to around 150 bar. The water is dosed with (amongst other things) LiOH to keep the conditions alkaline throughout so that general corrosion and the transportation of corrosion products are minimized [1]. Most of the research concerning the wear and corrosion behaviour of Stellites in PWR conditions has focused on Stellite 6 with much less in the literature relating to Stellite 3 . The research indicates that the high corrosion resistance of Stellite 6 in reactor environments is mainly due to the high chromium content of the alloy, which results in the formation of an oxide film with an enhanced chromium concentration [1]. When cast Stellite 6 was subjected to corrosion testing in high temperature, high pressure water replicating PWR conditions, x-ray photoelectron spectroscopy (XPS) revealed that the oxide layer formed was enriched in chromium and depleted in cobalt when compared to the matrix composition, indicating a preferential dissolution of cobalt at the oxide - solution interface [8]. It was argued that this is consistent with the significant solubility of cobalt and negligible solubility of chromium in these conditions. The formation and growth of oxide films in Stellite 6 in lithiated and borated high temperature water under reducing conditions has been attributed to a solid-state diffusion process due to the replacement of cobalt (outward diffusion) by oxygen (inward migration). Nevertheless, in the initial stages of oxidation before the formation of the protective oxide layer, the controlling factors are the kinetic reactions at the solution-surface interface [9-11]. Corrosion of UNS R30006 (similar to Stellite 6) in deaerated, $\mathrm{pH}$-adjusted water revealed that the primary oxide phase composition of the surfaces was $\mathrm{CoCr}_{2} \mathrm{O}_{4}$; some corrosion films were observed to have a high cobalt content on their outermost surface indicating that there may be an outer film of $\mathrm{CoFe}_{2} \mathrm{O}_{4}$ or $\mathrm{CoO}$. The corrosion films were thinner on the chromium-rich carbide phase than they were on the cobalt-rich matrix except 
where the carbide boundaries intersected the surface. Here, corrosion was observed to penetrate down and around the carbides and it was argued that this occurred due to chromium-depletion in the matrix material adjacent to the chromium-rich carbides, this depletion being associated with their precipitation [12]; however, it should be noted that no direct evidence of the chromium depletion in the matrix was presented.

Despite the use of Stellite 3 (or analogues) in PWR primary circuit components, there is a lack of detailed research in the published literature which examines the corrosion behaviour of these materials under such conditions. Accordingly, the present study seeks to examine the corrosion behaviour of WT- $3^{\circledR}$ in lithiated high temperature water with a focus on understanding the preferential corrosion reported in the literature by earlier workers.

\section{Experimental procedure}

\subsection{Materials}

The alloy used for this study was a cast cobalt-based alloy WT-3 (Weartech International Ltd, UK). The samples were discs of $60 \mathrm{~mm}$ diameter and a thickness of $3 \mathrm{~mm}$, machined from a $100 \mathrm{~mm}$ long bar. Table 1 shows the chemical composition of the alloy measured by optical emission spectroscopy provided by the manufacturer.

The WT-3 disc samples were as wet ground sequentially using silicon carbide papers, with the final size employed being 1200 grit. After grinding, the samples were polished using $6 \mu \mathrm{m}$ and $1 \mu \mathrm{m}$ diamond abrasive pastes.

\subsection{Pre-\& post autoclave exposure}

To investigate the corrosion mechanism of $\mathrm{WT}^{-} 3^{\circledR}$ in conditions replicating PWR environments, samples were exposed to hydrothermal conditions for 30 days in an 
autoclave with a volume of 1.22 litres, the vessel being constructed of 316 stainless steel. The samples were sat on stainless steel mesh shelves and immersed in a solution of lithiated water $(\mathrm{LiOH} 8.5 \mathrm{mg} / \mathrm{kg})$ with an initial $\mathrm{pH}$ of 10.5 . The test conditions were a temperature of $300^{\circ} \mathrm{C}$ and a pressure of 90 bar. After filling the vessel with the lithiated water and placing the samples and jigging into the vessel, the solution was deoxygenated by bubbling through for 90 minutes with oxygen-free nitrogen before valves were finally closed off; the residual oxygen in the solution was 3.06 ppm, measured to be with a Thermoscientific Orion Star A329 Portable meter. The autoclave was heated via an external electrical band heater to a temperature of $300^{\circ} \mathrm{C}$ (measured via a thermowell) and held at this temperature for 30 days whereupon the heater was turned off and the vessel and contents were allowed to cool naturally.

\subsection{Sample characterization}

\subsubsection{Optical microscopy}

Optical microscopy was performed with a NIKON UFX optical microscope. To enable the identification of the same regions of the microstructure before and after exposure, an indent was made on the sample using a macrohardness indenter to act as an identifiable marker.

\subsubsection{SEM}

Scanning electron microscopy (SEM) with energy dispersive X-ray analysis (EDX) was used for a detailed analysis of the microstructure. A JEOL 6490LV SEM was used at 20kV for back scattered electron (BSE) imaging; EDX point and linescan analyses were carried out to identify the chemical composition. 


\subsubsection{TEM}

Focused ion beam milling (FIB) was performed using an FEI Quanta 3D FIB-SEM. FIB sections were taken across the interfaces between both the Co-rich matrix and $\mathrm{M}_{7} \mathrm{C}_{3}$ and the Co-rich matrix and $\mathrm{M}_{12} \mathrm{C}$. FIB milling yielded thin foils with dimensions of $\sim 9$ $\mu \mathrm{m}$ by $5 \mu \mathrm{m}$ and a thickness of around $100 \mathrm{~nm}$. The thin foil lift-outs were transferred to JEOL 2100 TEM for STEM analysis. EDX linescans were used to measure the compositional profiles across interfaces of interest, whilst EDX mapping (with area quantification where appropriate) was also performed to allow the elemental distribution of the different elements in the thin foil to be observed.

\subsubsection{X-ray diffraction}

X-ray diffraction (XRD) was used to characterize the phases in the WT-3 sample both before and after autoclave exposure. XRD analysis was undertaken using a Siemens D500 diffractometer equipped with DACO-MP X-ray diffractometer which was operated at $40 \mathrm{kV}$ and $25 \mathrm{~mA}$ to generate $\mathrm{Cu}$ Ka radiation at a wavelength of $1.540 \AA$. The system was used in Bragg Brentano geometry, with a step size of $0.05^{\circ}$ and a step time of 4 seconds for a range of $2 \theta$ of $30-100^{\circ}$. Peak identification was done using Eva software using the PDF database from the International Centre for Diffraction data.

\subsubsection{Atomic force microscopy}

An Asylum Research (California, USA) MFP-3D atomic force microscope was used in contact mode to collect both height maps and electrical conductance maps of a polished surface of an as-received WT-3 sample. In conductive atomic force microscopy (CAFM), current passes through a sample via a conductive AFM tip. A current map of the sample surface was obtained under negative bias conditions. By 
mapping topography and current on a surface at the same time, cAFM facilitated the qualitative measurement of the electrical conductivity of the three main microstructural constituents of WT-3.

\section{Results}

\subsection{Microstructure and phase analysis}

Figure 1 shows a BSE image of the cast microstructure of the unexposed WT-3. The hypereutectic microstructure consists of a Cr-rich carbide phase (dark) and a W-rich carbide phase (bright) dispersed in a Co-rich matrix (mid-grey). Table 2 provides EDX analysis which shows the proportion of the main metallic elements present in each of these three phases (carbon is not included in the analysis). In accordance with previous research on an analogue alloy (Stellite 3), the XRD spectrum in Figure 2 confirms that the Cr-rich carbide phase has the $\mathrm{Cr}_{7} \mathrm{C}_{3}$-type structure [7], with the data in Table 2 indicating that the metallic element in this carbide is mainly chromium with a small amount of cobalt. Figure 2 indicates that the $\mathrm{W}$-rich carbide phase has the $\mathrm{Co}_{6} \mathrm{~W}_{6} \mathrm{C}$ structure, with the data in Table 2 indicating that tungsten, cobalt and chromium take the metallic sites in approximately equal proportions. The Co-rich matrix phase was shown to be a mixture of both f.c.c and h.c.p. structures (Figure 2) with a composition dominated by cobalt and chromium (Table 2). Also, it can be seen that no additional phases were found in the XRD spectrum after autoclave exposure of WT-3 (Figure 2), indicating that any corrosion products formed were too thin to be detected.

\subsection{Corrosion testing}

Figure $3 a \& b$ are optical microscope images of the same region on a WT-3 sample before and after autoclave exposure for 30 days at $300^{\circ} \mathrm{C}$. Figure $3 \mathrm{~b}$ shows there was 
a change in colour in the different phases; these colour changes are due to interference effects (similar to those observed in heat tinting) [13] and indicate that oxides of different thicknesses have formed on the different phases, suggesting a difference in the propensity for the three phases to corrode. Both the Cr-rich carbides and the $\mathrm{W}$-rich carbides had a blue colouration whilst the majority of the Co-rich matrix had a yellow-golden colour. In austenitic stainless steels oxides of different film thickness exhibit different colours, with yellow colours indicating thicknesses up to 75 $\mathrm{nm}$ and blues indicating thicknesses in the range $100-175 \mathrm{~nm}[14]$. Figures $3 c$ \& $\mathrm{d}$ show BSE images of the same surface region before and after autoclave exposure respectively, with Figure 4 presenting EDX elements maps for chromium, cobalt, tungsten and oxygen for the same region following autoclave exposure (corresponding to Figure 3d). In Figure 3d, a dark boundary layer was observed to have formed at the boundary between the $\mathrm{Cr}$-rich carbide phase and the Co-rich matrix phase, but not at the boundary between the $\mathrm{W}$-rich carbide and the Co-rich matrix, nor at the boundary between the Cr-rich carbide and the W-rich carbide. This boundary layer was confirmed to be oxygen-rich by EDX mapping (Figure 4d), indicating preferential oxidation between the Cr-rich carbide phase and the Co-rich matrix phase. Some morphological differences to the structure following autoclave exposure can also be seen on comparing Figures $3 c \& d$, indicating that there has also been general surface corrosion due to the autoclave exposure. Specifically, there has been loss of matrix phase (with an example highlighted in Figure $3 d$ ) with these regions leaving behind a residual oxide (as seen in Figure 4d); also, it can be seen that the morphology of the W-rich carbide phase has changed, indicating a general recession of this carbide phase which has exposed underlying regions of matrix phase. 
Figure 5 shows a bright field STEM image of a FIB-milled TEM foil of a cross-section through the interface region between a Cr-rich carbide phase and the Co-rich matrix phase following autoclave exposure, showing the penetration of the interfacial oxide to a distance of just less than $1 \mu \mathrm{m}$ below the surface. Morphologically, the shape of the oxide suggests that the oxide is being formed from the Co-rich matrix phase since the edge of Cr-rich carbide phase itself does not appear to have been altered by the formation of the interfacial oxide. The corresponding EDX maps are shown in Figures $5 b-g$ for a number of selected elements, giving an indication of their distribution across the three phases identified (the platinum map in Figure $5 \mathrm{~g}$ is presented to indicate the position of the platinum layer that was deposited for protection against sputtering during preparation of the FIB sample). The elemental maps indicate that the interfacial oxide is Cr-rich (Figure 5c), iron-rich (Figure 5d) and oxygen-rich (Figure 5e) but is depleted in the other metallic elements that were present in the Co-rich matrix, most notably cobalt (Figure 5b). The top surface oxide was also found to be Cr-rich (Figure 5c), iron-rich (Figure 5d) and oxygen-rich (Figure 5e), but was also Sirich (Figure 5f); it should be noted that this enhanced silicon level was not observed in the interfacial oxide. Figure 6 shows the STEM image again with regions and lines marked from which EDX data (excluding carbon) have been analysed. The data (atom fraction) from the five area regions are presented in Table 3; the interfacial oxide contains just over 50 at.\% oxygen, with approximately half of the metallic fraction being chromium but also with significant amounts of iron and cobalt. The interfacial oxide is similar to the oxide on top of the Co-rich matrix, except that the latter contains a much higher fraction of silicon. Figure $5 a$ indicates that the oxide that has grown on the Corich matrix is thicker than that which has grown on the Cr-rich carbide, with the compositions (Table 3) indicating that different types of surface oxides may have 
formed on the Co-rich matrix and the Cr-rich carbide, with the latter exhibiting much higher chromium levels.

Figure $7 \& 8$ presents EDX line scans (normalised across the metallic elements indicated) taken from the TEM-foil along the two lines shown in Figure 6, one of which crosses the boundary across the interfacial oxide (Figure 7) whilst the other crosses the boundary in a region where no oxidation has taken place (Figure 8). In the interfacial oxide (approximately $0.15-0.61 \mu \mathrm{m}$ from the start of the scan line in Figure 7), the $\mathrm{Cr}$ made up approximately 60 at. \% of the metallic elements, with $\mathrm{Fe}$ and $\mathrm{Co}$ making up 20 at.\% and 15 at.\% of the metallic fraction respectively. In Figure 8 where the EDX line scan crosses the interface between the Cr-rich carbide phase and Corich matrix phase in a region without corrosion, it can be seen that there is no enrichment or depletion of any metallic elements in either of the phases in the region close to the interface.

To determine the type of oxide formed, further milling of an autoclave-exposed Co-rich matrix - Cr-rich carbide interface cross-sectional sample was performed to obtain a very thin foil of $\sim 100 \mathrm{~nm}$ thickness suitable for selected area diffraction in the TEM. The milling resulted in loss of certain areas of the sample (including the surface), but a region which contained a small amount of the interfacial oxide was preserved. Figure 9a shows the high-resolution STEM image of the region examined, with Figure 9b showing the oxygen EDX map of the sample, with the interfacial oxide being indicated by the region of highest oxygen concentration. Table 4 shows the chemical composition of this fragment of interfacial oxide measured by EDX analysis, which indicates a similar composition to that presented in Table 3. To determine the crystal structure of the oxide, a selected area diffraction pattern was taken from within the oxide region. Figure $9 \mathrm{c}$ shows the selected area diffraction pattern from the oxide 
which exhibits a ring structure (indicating a very fine-grained oxide) with the rings being indexable to the $\mathrm{M}_{3} \mathrm{O}_{4}$ crystal structure. Three rings have been indexed as indicated in Table 5, yielding a lattice parameter of $8.584 \mathrm{~nm}$; this compares favourably with the lattice parameter for $\mathrm{CoCr}_{2} \mathrm{O}_{4}$ of $8.364 \mathrm{~nm}$ (JCPDS file number 00-022-1084)[15], with this basic composition being a reasonable match to the $\mathrm{Co}: \mathrm{Cr}$ ratio indicated by the EDX analyses (Tables 3 and 4). The calculated lattice parameter is slightly larger than that of the pure $\mathrm{CoCr}_{2} \mathrm{O}_{4}$ spinel due to the presence of other elements in the spinel [12].

The BSE-SEM image of the surface following autoclave exposure (Figure 3d) along with the associated EDX mapping (Figure 4d) indicated that there was no preferential oxidation at the interface between the $\mathrm{W}$-rich carbide phase and the Co-rich matrix phase. This interface was therefore characterized via TEM following milling of a foil $370 \mathrm{~nm}$ thick across this interfacial section. Figure 10a is the dark field STEM image of the cross-section across the two phases. Figures 10b - $\mathrm{h}$ show the EDX maps of the elements as indicated. The EDX Cr map (Figure 10c) indicates that there were some localised areas at the boundary that had a high chromium concentration, although this was not observed throughout the whole interface between the W-rich carbide phase and the Co-rich matrix phase. Figure 11 shows the STEM image, again with regions and lines marked from which EDX data (excluding carbon) have been analysed. The data (atom fraction) from the two area regions are presented in Table 6 whilst Figure 12 presents the EDX line scan (normalised across the metallic elements indicated only) taken from the TEM-foil along the line shown in Figure 11; again, no clear preferential enhancement or depletion of any elements are seen in either the Co-rich matrix or in the W-rich carbide close to the interface. 
In order to give an indication as to whether the preferential interfacial corrosion had any electrochemical nature to it, conductive atomic force microscopy (cAFM) was conducted on a polished region of an unexposed sample which contained the three phases, so that their electrical conductivity could be qualitatively compared. Figure 13a shows an optical image of the region where the cAFM was conducted, with Figures $13 b$ \& c showing the height (profile) map and current map respectively. Figure 13d shows a BSE-SEM image of the same area, which allows the nature of the three phases to be readily identified as before.

The height differences following polishing of a flat surface give some indication of the tendency to resist polishing (and therefore some indication of the relative wear resistance of each of the three phases). Comparison of Figure 13b and Figure 13d shows that there is good correlation between the height map and the microstructure; the high points in the profile are related to the Cr-rich carbide, with the $\mathrm{W}$-rich carbide generally being at intermediate height and the Co-rich matrix generally exhibiting the lowest points in the profile map. This gives some qualitative indication that the $\mathrm{Cr}$-rich carbide is the most wear resistant of the three phases, with the matrix exhibiting the lowest wear resistance.

For current mapping in the AFM imaging, a negative bias was used to collect the current to avoid local anodic oxidation of the tip. The current map in Figure 13c shows that the material allowed different currents to be conducted at various points across the map, and comparison with Figure 13d indicates that this correlates well with the microstructural makeup; the highest current flowed in the Co-rich matrix, followed by that in W-rich carbide phase with the lowest current being carried in the $\mathrm{Cr}$-rich carbide phase. However, the CAFM data does indicate significant conductivity of both carbide types, which may lead to electrochemically enhanced corrosion. 


\section{Discussion}

The major phase in WT-3 is the Co-rich matrix phase (both f.c.c and h.c.p), with f.c.c being the main phase as indicated by the XRD spectrum in Figure 2 where the peak with the highest intensity was the cobalt f.c.c peak at $51^{\circ}$. In most cases, cobalt-based solid solutions exist in a metastable f.c.c. form at room temperature unless they have been subjected to mechanical deformation [16]. During the solidification of Stellites, large quantities of carbides form in the microstructures; cast samples have larger matrix areas between the carbide dendrites that are unprotected by the harder carbides, which have a coarse size (coarse carbide size of $5-20 \mu \mathrm{m}$ ). The large carbide size in the cast WT-3 indicates slow cooling of the casts. Image analysis of the cast WT-3 (using ImageJ) indicates that it has approximately 26 vol\% of carbides (Cr-rich carbides \& W-rich carbides together).

After autoclave exposure, optical microscopy revealed that both carbide types exhibited a blue colour whilst the Co-rich matrix exhibited a golden yellow colouration (a similar colouration of the Co-rich matrix phase was seen in a similar test on the corrosion of wrought Stellite 6 [11]). This colouration indicates that the corrosion films are very thin, but that the film formed on the carbides are thicker than that formed on the Co-rich matrix. This is in contrast to the data from the STEM imaging (Figures $5 a$ \& 6) where the oxide on the carbide appears thinner than the oxide on the Co-rich matrix. The general recession of the $\mathrm{M}_{7} \mathrm{C}_{3}$ and the $\mathrm{M}_{12} \mathrm{C}$ indicated by comparison of Figure $3 c$ and Figure $3 d$ give clear indication that the carbides themselves are corroding, along with the Co-rich matrix. From Figure 5c, the surface of the cobaltmatrix seems to have receded more than the surface of the carbide, although it is recognised that the differential heights may have existed before the corrosion testing 
(see Figure 13b). The recession of the surface occurred by general corrosion causing the loss of the Co-rich matrix phase by the selective dissolution of cobalt leaving behind an oxide film.

Alongside this general corrosion, enhanced corrosion was seen at the interface between the $\mathrm{Cr}$-rich carbide $\left(\mathrm{M}_{7} \mathrm{C}_{3}\right.$-type) and the Co-rich matrix. This oxide has been shown to have penetrated to a depth of about $1 \mu \mathrm{m}$ below the surface following the 30 days autoclave exposure, and has been shown to be a spinel of the $\mathrm{CoCr}_{2} \mathrm{O}_{4}$-type (i.e. a chromite). It is also notable that this interfacial oxide occurs only at interfaces of this type, and not at the other interfaces. The chromite could be formed by the reaction between cobalt oxide and chromium oxide according to the reaction below.

$$
\mathrm{CoO}+\mathrm{Cr}_{2} \mathrm{O}_{3} \rightarrow \mathrm{CoCr}_{2} \mathrm{O}_{4}[17]
$$

It is proposed that the interfacial oxide forms from corrosion of the Co-rich matrix in this region (as opposed to being a corrosion product of the carbide itself). The relative proportions of just the metallic elements in both the Co-rich matrix and the interfacial oxide are shown in the EDX line scan in Figure 7; it can be seen that (compared to the matrix material from which it is derived), the interfacial oxide has significantly enhanced levels of chromium and iron, with cobalt being significantly reduced. The reduction in the fraction of cobalt amongst the metals in the interfacial oxide indicates that there was preferential dissolution of cobalt from the Co-rich matrix with loss of cobalt ions to the lithiated water solution. Similar selective dissolution of cobalt from cobalt - chromium alloys has been previously observed under neutral or acidic aqueous conditions [9]. In addition, it has been shown that cobalt is considerably more soluble than chromium in lithiated high temperature water under reducing conditions [11]. The reasons for the increase of the iron content in the interfacial oxide is less easy to explain, but it is noted that the autoclave itself and the shelf furniture within it 
are constructed of stainless steel, and the oxide may have been in part affected by deposition from solution.

When a similar preferential oxide formation between $\mathrm{M}_{7} \mathrm{C}_{3}$ and the Co-rich matrix has been observed previously in Stellite $6[12,18]$, it has been assumed (without any direct evidence) that this was due to chromium depletion in the matrix in the vicinity of the Cr-rich carbide which then led to preferential corrosion in this area (similar to the sensitization effect that is observed in stainless steels). However, it has been shown in this work that no region of depleted chromium exists in the Co-rich matrix close to the $\mathrm{Cr}$-rich carbide (Figure 8). Alongside this preferential corrosion at the $\mathrm{M}_{7} \mathrm{C}_{3}-\mathrm{matrix}$ boundary, it is noted that there is no preferential corrosion of the matrix at the $\mathrm{M}_{12} \mathrm{C}$ matrix boundary. In a similar way to the boundary between the matrix and the $\mathrm{M}_{7} \mathrm{C}_{3}$, no significant enhancement or depletion of any elements are observed in the matrix close to the $\mathrm{M}_{12} \mathrm{C}$ boundary (Figure 12). As such, it is concluded that the enhanced corrosion in the matrix close at the boundary with the $\mathrm{M}_{7} \mathrm{C}_{3}$ is not driven by changes in the alloy makeup, and therefore it is proposed that the main alternative is that this is an electrochemical effect.

For an electrochemical effect, the phases in question need to be electrically conducting. The literature indicates that the Co-rich matrix phase has an electrical conductivity of $17.9 \mathrm{MS} \mathrm{m}^{-1}[19]$ with the electrical conductivity of $\mathrm{Cr}_{7} \mathrm{C}_{3}$ also being relatively high, being around only a twentieth that of the metallic matrix itself $(0.91 \mathrm{MS}$ $\mathrm{m}^{-1}$ ) [20]. Although no data relating to the electrical conductivity of the $\mathrm{M}_{12} \mathrm{C}$ was available in the literature, Figure $13 \mathrm{c}$ qualitatively indicates that the $\mathrm{M}_{12} \mathrm{C}$ has an electrical conductivity somewhere between that of the Co-rich matrix and the Cr-rich $\mathrm{M}_{7} \mathrm{C}_{3}$. It is therefore proposed all the phases in the system have electrical conductivities which would support electrochemical effects. 
In considering the behaviour of Stellite 6 in a chloride containing environment, it has been argued that the carbides $\left(\mathrm{M}_{23} \mathrm{C}_{6}\right.$ and $\left.\mathrm{M}_{7} \mathrm{C}_{3}\right)$ are more noble than the metallic matrix, and thus can promote breakdown of the normal passive form and result in corrosion [2]. It is argued in the current work that this breakdown is promoted by the $\mathrm{M}_{7} \mathrm{C}_{3}$-type carbide, but not by the $\mathrm{M}_{12} \mathrm{C}$-type carbide, and that this is due to the differences in the potential of the two carbide types. Literature values of the standard Gibbs free energy of formation of the two types of carbides: $\mathrm{M}_{12} \mathrm{C}$-type carbide is $\Delta^{\circ} \mathrm{G}=$ $-130 \mathrm{~kJ} \mathrm{~mol}^{-1}$ at $1150^{\circ} \mathrm{C}[21]$ and the $\mathrm{M}_{7} \mathrm{C}_{3}$-type carbide is $\Delta^{\circ} \mathrm{G}=-226 \mathrm{~kJ} \mathrm{~mol}^{-1}$ at $1127^{\circ} \mathrm{C}$ [22]. Comparing the literature values of the standard Gibbs free energy of the $\mathrm{M}_{12} \mathrm{C}$ type carbide and the $\mathrm{M}_{7} \mathrm{C}_{3}$-type carbide, the latter has a more negative Gibbs free energy, therefore set up a greater potential than the $\mathrm{M}_{12} \mathrm{C}$-type carbide against the Corich matrix. The higher electrical potential between the $\mathrm{M}_{7} \mathrm{C}_{3}$-type carbide against the Co-rich matrix enhanced the electrochemical corrosion between these two phases.

\section{Conclusion}

General surface corrosion led to loss of the Co-rich matrix phase by the selective dissolution of cobalt leaving behind an oxide film and there was also general recession of the W-rich carbide phase which revealed the underlying regions of the matrix phase.

A thin oxide film was formed on the surface which was non-detectable by XRD. A thicker oxide film was observed over the Co-rich matrix compared to the Cr-rich carbide.

There was interfacial corrosion between the Cr-rich carbide phase and the Co-rich matrix. The preferential oxide observed between the $\mathrm{M}_{7} \mathrm{C}_{3}$ and the Co-rich matrix is due to an electrochemical effect. All the phases are electrically conductive, and the AFM current mapping showed decreasing order of Co-rich matrix, W-rich carbide and 
Cr-rich carbide. No interfacial corrosion was found between the Co-rich matrix and carbides which are rich in either $\mathrm{Cr}, \mathrm{W}$ or $\mathrm{Cr}$.

\section{Acknowledgements}

The authors are grateful for the financial support from Rolls Royce plc for support of a studentship for GNK. Michael Fay, Chris Parmenter and Thomas James (all colleagues from the University of Nottingham) are acknowledged for the assistance and advice that they provided in support of the experimental work presented here.

\section{Data Availability statement}

All the data generated or analysed in this study are included in this published article.

\section{References}

[1] D. Féron, - Overview of nuclear materials and nuclear corrosion science and engineering, in: D. Féron (Ed.), Nuclear Corrosion Science and Engineering, Woodhead Publishing, Cambridge, 2012, pp. 31-56.

[2] F. Rosalbino, G. Scavino, Corrosion behaviour assessment of cast and HIPed Stellite 6 alloy in a chloride-containing environment, Electrochimica Acta 111(Supplement C) (2013) 656-662.

[3] H. Yu, R. Ahmed, H. de Villiers Lovelock, A comparison of the tribo-mechanical properties of a wear resistant cobalt-based alloy produced by different manufacturing processes, Journal of tribology 129(3) (2007) 586-594.

[4] S. Zhang, D. Zhao, Aerospace Materials Handbook, CRC Press, Boca Raton, USA, 2012.

[5] W.L. Silence, Effect of Structure on Wear Resistance of Co-, Fe-, and Ni-Base Alloys, Journal of Lubrication Technology 100 (3) (1978) 428-435.

[6] J.R. Davis, A.S.M.I.H. Committee, Nickel, Cobalt, and Their Alloys, ASM International, Materials Park,Ohio,USA, 2000.

[7] R. Liu, J.H. Yao, Q.L. Zhang, M.X. Yao, R. Collier, Sliding wear and solid-particle erosion resistance of a novel high-tungsten Stellite alloy, Wear 322-323 (2015) 4150. 
[8] C. Maffiotte, M. Navas, M.L. Castaño, A.M. Lancha, XPS characterization of oxide films formed in cobalt-based alloys during corrosion tests at high temperature, Surface and Interface Analysis 30(1) (2000) 161-166.

[9] N.S. McIntyre, D. Zetaruk, E.V. Murphy, X-Ray photoelectron spectroscopic study of the aqueous oxidation of stellite-6 alloy, Surface and Interface Analysis 1(4) (1979) 105-110.

[10] W. Hocking, D. Lister, Corrosion of Stellite-6 in Lithiated and Borated Hightemperature Water, Surface and Interface Analysis 11 (1988) 45-59.

[11] W.H. Hocking, F.W. Stanchell, E. McAlpine, D.H. Lister, Mechanisms of corrosion of stellite-6 in lithiated high temperature water, Corrosion Science 25(7) (1985) 531557.

[12] M.J. LeClair, J.T. Orr, M.C. Burrell, P.C. Sander, R.A. Morris, Corrosion and Corrosion Product Release Behavior of Co-Cr-W-C Weld Deposit, Nace International corrosion conference \& expo 2015, NACE International, 2015, pp. 1-15.

[13] G.F. Vander Voort, Metallography, Principles and Practice, ASM International, New York,USA, 1984.

[14] M. Somervuori, L.S. Johansson, M.H. Heinonen, D.H.D. van Hoecke, N. Akdut, H.E. Hänninen, Characterisation and corrosion of spot welds of austenitic stainless steels, Materials and Corrosion 55(6) (2004) 421-436.

[15] JCPDS, International Centre for Diffraction Data® http://www.icdd.com/, 1997 2017 (accessed 20/11/2017.2017).

[16] J.W. Martin, Concise Encyclopedia of the Structure of Materials, Elsevier Science, University of Oxford,UK, 2006.

[17] C.E. Lowell, D.L. Deadmore, Formation and breakdown of a protective layer of chromium sesquioxide on L-605 alloy at 1100 degrees C, Lewis Research Center,NASA, Cleveland, Ohio,US, 1973, pp. 1-19.

[18] H.E. Placko, S.A. Brown, J.H. Payer, Effects of microstructure on the corrosion behavior of $\mathrm{CoCr}$ porous coatings on orthopedic implants, Journal of Biomedical Materials Research 39(2) (1998) 292-299.

[19] G.W.C. Kaye, T.H. Laby, Tables of Physical and Chemical Constants 14 ed., Longman, London, 1973.

[20] M.S. Koval'chenko, V.V. Ogorodnikov, Effect of neutron irradiation on the electrical resistances of titanium and chromium carbides, Soviet Atomic Energy 21(4) (1966) 974-976.

[21] T. Johansson, B. Uhrenius, Phase equilibria, isothermal reactions, and a thermodynamic study in the Co-W-C system at $1150^{\circ} \mathrm{C}$, Metal Science 12(2) (1978) 83-94.

[22] L.B. Pankratz, U.S.B.o. Mines, Thermodynamic Properties of Carbides, Nitrides, and Other Selected Substances, U.S. Department of the Interior, U.S. Bureau of Mines, USA, 1995. 